\title{
Frontières
}

\section{Fichiers MP3 se retrouvant sur le CD qui accompagne ce numéro de Frontières}

Volume 20, numéro 2, printemps 2008

Les musiques et la mort

URI : https://id.erudit.org/iderudit/018371ar

DOI : https://doi.org/10.7202/018371ar

Aller au sommaire du numéro

Éditeur(s)

Université du Québec à Montréal

ISSN

1180-3479 (imprimé)

1916-0976 (numérique)

Découvrir la revue

Citer ce document

(2008). Fichiers MP3 se retrouvant sur le CD qui accompagne ce numéro de Frontières. Frontières, 20(2), 128-128. https://doi.org/10.7202/018371ar d'utilisation que vous pouvez consulter en ligne.

https://apropos.erudit.org/fr/usagers/politique-dutilisation/ 


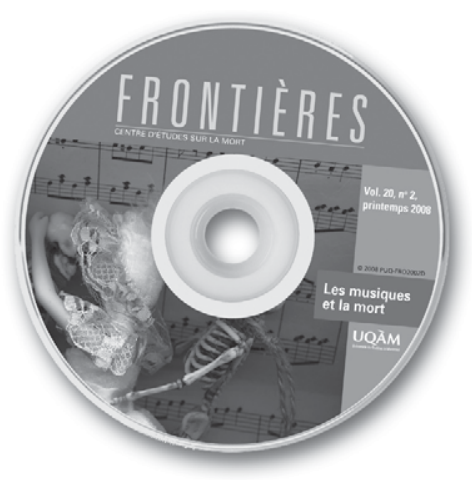

In the blindage, chant soviétique.

Musique : K. Listov ; paroles : A. Surkov ; 1942.

Durée : $2 \min 58 \mathrm{~s}$

Dies Irae du Requiem en ut mineur de Luigi Cherubini, interprété par le Chœur de I'UQAM et l'Orchestre de la Société philharmonique de Montréal; direction: Miklós Takács, disque CHORAM.

Durée : $9 \min 31 \mathrm{~s}$

II bianco e dolce cigno (La mort du cygne) de Jacques Arcadelt et Alfonso D'Avalos (1539). Madrigal italien interprété par The Hilliard Ensemble (Lynne Dawson, Gillian Fisher, Rogers Covey-Crump, Paul Hillier), Londres, EMI 1988.

Durée : $1 \min 59 \mathrm{~s}$

Noolo exécuté pour les funérailles de Koffi Brou, à Kangandissou (pays anyi, en Côte-d'Ivoire), dans l'aprèsmidi du 5 décembre 1996.

Durée : $7 \min 16 \mathrm{~s}$

Làngnìn des cantateurs lors de funérailles au Burkina Faso en février 2006. Xylophone dègàar paire de baguettes frappées, membranophone sur calebasse et groupe de cantateurs. Enregistrement : D. Vaulay.

Durée : $9 \min 14 \mathrm{~s}$

Miroloi Albasan, extrait de la plage 1 du disque Grèce, Koumpania Xalkias, Musiciens traditionnels d'Épire, collection Inédit Maison des Cultures du Monde, W260117, 2004.

Durée : $4 \min 20 \mathrm{~s}$

Lamentation des Yézidis d'Arménie. Lamentation féminine improvisée par Hasmig à la demande de l'ethnomusicologue, en avril 2007, au village de Rya Teze, dans la région d'Aparan, en Arménie. Cette lamentation parle de la mort de son fils et de son frère. Au fil de la lamentation, Hasmig les compare à des cerfs, décrit leur corps vaillant et leur beauté et évoque sa souffrance de mère et de sœur, son malheur, sa malchance. Les pleurs et gémissements audibles dans l'enregistrement sont ceux de la mère de Hasmig. Enregistrement : $\mathrm{E}$. Amy de la Bretèque.

Durée : $7 \min 28 \mathrm{~s}$

Çeko, lamentation des Yézidis d'Arménie. Lamentation enregistrée en studio, composée et chantée par Jono Temuryan. Accompagnement au duduk (type de hautbois en bois d'abricotier) et synthétiseur. Cette lamentation est dédiée à Çeko Xidir, chef de gang très connu dans le monde criminel, tué à Moscou en 1994 à l'âge de 26 ans.

Durée : $5 \min 41 \mathrm{~s}$

Veillée funèbre tsigane pour Haritcka, quartier tsigane de Ceuaş, en Transylvanie (Roumanie), le 31 mai 2004, vers 23 heures. Deuxième nuit de veillée funèbre pour Haritcka, morte à l'âge d'environ 70 ans, dans sa maison. Les musiciens, pour la plupart parents de la défunte, jouent une suite instrumentale qui se superpose aux pleurs des neamuri, la famille élargie. La première moitié

de la suite est composée d'airs de jale, de chagrin: doina ou kerservesek (en rythme non mesuré), et meseliekri (mélodies " de table ", en rythme lent, légèrement asymétrique); la deuxième moitié comprend des mélodies de danse (csárdás et hâr ag).

Violon électrique: lambor Ferenc "Tocsila"; contr (alto à trois cordes): Mezei Ferenc "Cangalo"; accordéon (touches bouton): Alexandra Beaujard "Alex»; accordéon (touches piano) : "Buciurica "; contrebasse (à trois cordes, jouée à I'archet): Mezei Poli.

Durée : $16 \min 42 \mathrm{~s}$

Prologue de La Cantate de Bisesero de Garett List, GROUPOV, spectacle Rwanda 94, 2001. Garett List, composition, direction et trombone; Manuela Bucher, violon; Fabian Fiorini et Geneviève Foccroule, piano; Vincent Jacquemin, clarinette ; Véronique Lierneux, violon; Marie-Ėve Ronveaux et Jean-Pol Zanutel, contrebasse ; Christine Schaller, Véronique Sonck, chant; Jean-Marie Muyango, chant et percussions; Augustin Majyambere, percussions en solo ; Massamba, chant, cithare, percussions; Dorcy Rugamba, percussions; Younouss Diallo, acteur et narrateur ; Stéphane Fauville, acteur et narrateur; Carole Karemera, actrice et narratrice; Jeanne Kayitesi, actrice et narratrice; Francine Landrain, actrice et narratrice; Joëlle Ledent, actrice et narratrice; Max Parfondry, acteur et narrateur; François Sikivie, acteur et narrateur.

Durée : $3 \min 44 \mathrm{~s}$

Es brent (It is burning). Compositeur: Mordechai Gebirtig (1877-1942). Interprète: Yves Markowicz. Cette chanson fut écrite en 1938, en réponse à un pogrom qui eut lieu dans la ville de Przytyk, en Pologne, en 1936. En voici un extrait: "Brûle, frères, il brûle!/Oh, notre pauvre village, hélas, brûle!/Les vents mauvais avec leurs rafales/Déchirent, brisent et dispersent,/

Les flammes sauvages sont de plus en plus fortes/ Tout autour, tout brûle déjà. "

Durée : $2 \min 25 \mathrm{~s}$

Le funambule et la trapéziste. Paroles et musique de la chanson: Milly. Arrangement: Valentin Akriche.

Durée : $4 \min 11 \mathrm{~s}$

L'orée sur le grand silence..., lecture par Jean-François Casabonne, le 21 mars 2005, lors du cinquième et dernier récital de Pierre Jasmin pour sa série Mozart, à la salle Pierre-Mercure du Centre Pierre-Péladeau.

Durée : $5 \min 42 \mathrm{~s}$

Adagio en si mineur Köchel 540 de Mozart, interprété par Pierre Jasmin, à la salle Pierre-Mercure du Centre Pierre-Péladeau, le 21 mars 2005. Enregistrement: Sylvain Arseneault, studio audiovisuel de I'UQAM.

Durée : $9 \min 34 \mathrm{~s}$

$2^{e}$ mouvement, Concertino de Saint-Irénée. Compositeur: François Dompierre, 1984. Fuvre dédiée à Françoys Bernier.

Durée : $6 \min 4 \mathrm{~s}$ 